\title{
EDITORIAL
}

\section{How should we assess patent foramen ovale?}

\section{Seiler}

Heart 2004;90:1245-1247. doi: 10.1136/hrt.2003.031500

\section{Patent foramen ovale is being increasingly linked to a number of pathological conditions, most recently the prevalent disorder of migraine with aura. Many experts now no longer regard PFO as a harmless pimple but more a peril to health and even longevity}

r n 1877, Cohnheim first indicated a causal relation between patent foramen ovale (PFO) and relevant sickness when analysing the case of a young woman with a stroke. ${ }^{1}$ Since then, PFO has been recognised as a potential conduit for paradoxical embolism of thrombus, fat, air, or "things" as harmless as desaturated blood leading to various ailments, such as cerebral ischaemia, ${ }^{2}$ transient global amnesia, decompression illness in divers, ${ }^{3}$ refractory hypoxemia in the presence of right ventricular infarction or severe pulmonary disease, and the rare platypnoea-orthodeoxia syndrome. PFO has even been recently linked to the prevalent disorder of migraine with aura, ${ }^{45}$ and now many experts no longer regard it as a harmless pimple but as a peril to health and even longevity. Thus, the drastic statement can be envisaged that any hole, maybe except for valvar gaps and ventricular or coronary lumens, should be closed as soon as it is detected. Aside from debating the latter mentioned visions, the main goal of this article is to provide a practical overview of how a PFO can be identified best.

\section{RELEVANCE AND PREVALENCE}

In contrast to the notion of an abridged life after birth due to an open foramen ovale, the negative effect of a closed foramen ovale before birth is not speculative. The function of the foramen ovale before birth is that well oxygenated blood from the inferior vena cava bypasses the not yet operational pulmonary circulation. In this context, the Eustachian valve causes the blood to stream towards the foramen ovale and directly into the left atrium. During and shortly after birth, vascular resistance in the pulmonary circulation is lowered and resistance in the systemic circulation is heightened, which leads to a net increase in left atrial pressure causing the "door" of the left sided septum primum to close against the "frame" of the right sided septum secundum. Observed over the "first ten decades of life", 6 the door is glued to the frame in $73 \%$ of people-that is, PFO occurs in $27 \%$. According to the study by Hagen and colleagues, ${ }^{6}$ the prevalence of PFO found at necropsy decreases with age from $34 \%$ in the first to $22 \%$ in the 10th decade of life. ${ }^{6}$ Based on this single study finding, it could be wildly speculated that people with PFO die earlier than those without, which would cause the prevalence of PFO in older versus younger people to drop.

Compared to the hypothesis just mentioned, an association between PFO and cryptogenic stroke or peripheral systemic emboli as well as decompression illness, together with ischaemic brain lesions in divers, has been well established. ${ }^{3}{ }^{7} \mathrm{~A}$ beneficial effect on recurrent cerebral stroke or transient ischaemic attack of transcutaneous PFO device closure over anticoagulant or antiplatelet treatment has been indicated, ${ }^{8}$ although no prospective, controlled clinical trial has yet confirmed this result. There is practically no or only very preliminary evidence that PFO device closure is capable of preventing ischaemic brain lesions and decompression illness in divers, or that it "cures" migraine with aura. ${ }^{910}$

\section{GENETIC ASPECTS}

Considering the association between migraine with aura and occurrence of PFO, as well as the presence of a family history in the majority of patients with migraine, it is reasonable to hypothesise that genetic constellations may influence the existence of a PFO and that it may be inherited. The recent study by Rodriguez and colleagues ${ }^{11}$ supports this notion only in part by documenting a similar frequency of PFO among race-ethnic groups, but a higher prevalence of right atrial anatomy favouring paradoxical embolism (prominent Eustachian valve) and more often a large PFO in whites and Hispanics compared with black patients who had suffered an ischaemic stroke.

The strength of the work by Wilmshurst and colleagues $^{12}$ presented in the current issue of Heart lies in the high originality of its content by asking for the first time whether PFO is inherited into adulthood (after all, it is $100 \%$ inherited at birth because it closes only immediately after delivery). Accordingly, the really interesting part of the study is the finding that in 16 of 20 families of the respective index patient with a large right-to-left atrial shunt, a similar shunt could also be found. The second part of the investigation, where the relation of the shunt to migraine is examined, is less enthralling. The authors firmly state that "there is an association between migraine with aura and right-to-left shunts", 12 and that only large shunts are related to migraine with aura. The cited study to support this statement as well as the paper presented in this issue contain one conceptual flaw-that is, the method used to detect right-to-left shunts

Abbreviations: PFO, patent foramen ovale; TOE, transoesophageal echocardiography; TTE, transthoracic echocardiography 
(transthoracic echocardiography (TTE)) is too blunt to discern the entire range of shunts. Thus, it may be correct that large shunts are the only ones relevant for any disease manifestation, but this cannot be deduced on the basis of transthoracic or even transcranial ultrasound, because small and moderate shunts remain undetected (up to a third ${ }^{13}$ ) or artefacts in the left atrium may be interpreted as such.

\section{DIAGNOSIS}

Considering the mentioned associations between certain disorders and an increased prevalence of PFO, there is approximately a $50 \%$ chance of finding a $\mathrm{PFO}$ in a patient younger than 50 years of age if she or he had suffered a cryptogenic stroke, an event of decompression illness during a dive, or episodes of migraine with aura. Using purely clinical means, it is not possible to suspect or even diagnose a PFO. At the low end of technological means, transcutaneous oxymetry can be employed to find a right-to-left shunt by detecting a fall in oxygen saturation immediately after release of a Valsalva manoeuvre. ${ }^{14}$ In comparison, transcranial, contrast enhanced Doppler ultrasound detects right-to-left shunts more sensitively, but it is unable to locate the "hole" as with echocardiography, a diagnostic feature which is essential for subsequent treatment of the disorder.

The gold standard for diagnosing PFO is contrast enhanced transoesophageal echocardiography (TOE) (fig 1). At our institution, echo contrast consists of an ad hoc sonicated mixture of $0.2 \mathrm{ml}$ of air and $1.8 \mathrm{ml}$ of a gelatine containing plasma expander (1:9 mixture). However, TOE is only superior to TTE if certain conditions are fulfilled and pitfalls related to TOE are known.

First, the correct use of the Valsalva manoeuvre to provoke a right-to-left shunt is essential. Using TOE in a patient with a strong gag reflex who has to be heavily sedated may spoil his ability to cooperate to such an extent that an adequate Valsalva manoeuvre is no longer possible. The Valsalva
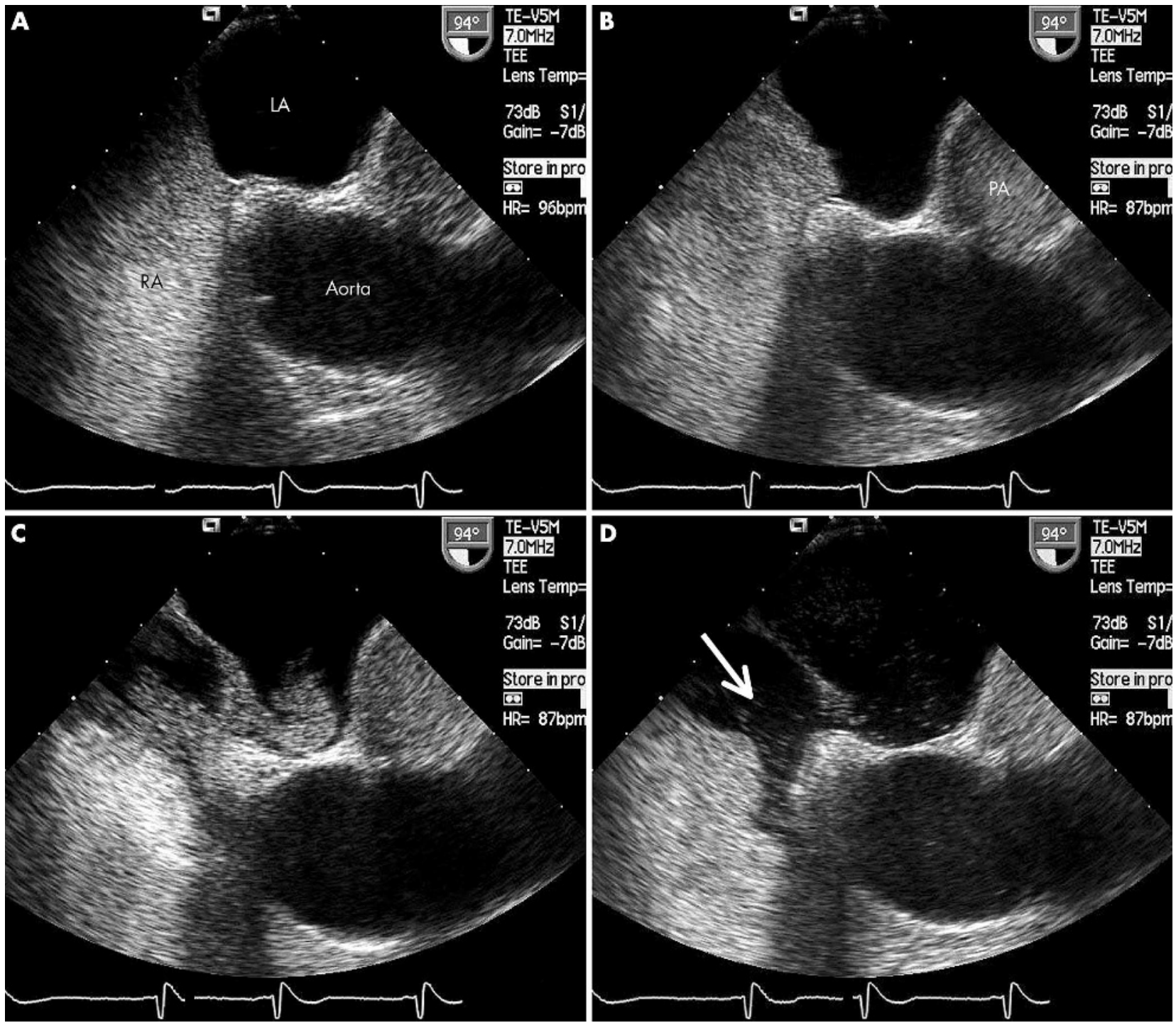

Figure 1 Transoesophageal contrast echocardiography (TOE) for the detection of patent foramen ovale (PFO). (A) TOE long axis view (right sidecranial; left side-caudal) showing the left atrium (LA) and the aortic root free of ultrasound contrast medium as well as the right atrium (RA) filled with contrast bubbles. The image is taken close to the end of the Valsalva strain phase with the interatrial septum bulged towards the RA. (B) Identical TOE image plane as in all other panels (long axis view) taken immediately after release of the Valsalva strain phase: the interatrial septum (fossa ovalis region) now bulges towards the LA, thus indicating a pressure rise in the RA above that in the LA (PA, pulmonary artery). (C) Long axis view image obtained instantaneously after that in panel B revealing a shunt of contrast medium across a PFO from the right to the left atrium. (D) The shunt is much less pronounced on this next image (PFO grade 3). Washout of contrast medium in the RA is visible (arrow), which is caused by the inflow of contrastfree blood from the inferior vena cava. 
manoeuvre is most efficiently carried out as follows: it should be practised before the start of either TOE or TTE; it ought to be started without prior inspiration; and it has to last about 5-10 seconds-that is, until the first bubbles of echocontrast medium appear in the right atrium.

Second, if the Valsalva manoeuvre is not carried out properly, the patient should be asked to cough 2-3 times.

Third, and assuming a left lateral supine position of the patient, the enhanced volume supply to the right atrium during the release phase of the Valsalva manoeuvre can be supported by injecting echo contrast from the right cubital vein and by lifting this arm at the end of the manoeuvre. Conversely, flow of contrast by injection from the left arm, which may even support the patient's head, can be very much impeded. A good quality of the Valsalva manoeuvre should be ascertained by observing a change in the convexity of the fossa ovalis membrane from right to left (fig 1A, B).

Fourth, TOE's advantage of direct imaging of and focusing on the site of the PFO should be exploited (optimally seen in a longitudinal plane; fig 1 ).

Fifth, the appearance of contrast bubbles in the left atrium (fig IC) occurs within 3-4 heart beats after release of the Valsalva manoeuvre; by TOE, it can be recognised that the structure of the bubbles is altered when they cross the lung (less echo dense, smaller).

Sixth, washout of contrast bubbles by flow from the inferior vena cava streaming alongside a prominent Eustachian valve (fig lD) can be so dynamic that they are not reaching the site of the PFO. In such cases, a higher volume of contrast ( $5 \mathrm{ml}$ instead of $2 \mathrm{ml}$ ) can be given to overcome the problem or the contrast injection should be performed from a vein in the foot.

Finally, the size of the PFO is estimated only using TOE by a score of $0-3$, with a score of 1 representing the crossover of a few single bubbles, and a score of 3 representing the shunt of an entire cloud of bubbles (score 2 between 1 and 3 ).

\section{REFERENCES}

1 Cohnheim J. Thrombose und Embolie. In: Cohnheim J, ed. Vorlesung über allgemeine Pathologie. Berlin: Hirschwald, 1877:134.

2 Lechat $P$, Mas JL, Lascault G, et al. Prevalence of patent foramen ovale in patients with stroke. N Engl J Med 1988;318:1148-52.

3 Schwerzmann M, Seiler C, Lipp E, et al. Relation between directly detected patent foramen ovale and ischemic brain lesions in sport divers. Ann Intern Med $2001 ; 134: 21-4$.

4 Wilmshurst PT, Nightingale S. Relationship between migraine and cardiac and pulmonary right-to-left shunts. Clin Sci 2001;100:215-20.

5 Schwerzmann M, Nedeltchev $K$, Lagger F, et al. Patent foramen ovale and migraine with aura [abstract]. J Am Coll Cardiol 2003;41:464A.

6 Hagen PT, Scholz DG, Edwards WD. Incidence and size of patent foramen ovale during the first 10 decades of life: an autopsy study of 965 normal hearts. Mayo Clin Proc 1984;59:17-20.

7 Di Tullio M, Sacco RL, Gopal A, et al. Patent foramen ovale as a risk factor for cryptogenic stroke. Ann Intern Med 1992;117:461-5.

8 Windecker S, Wahl A, Nedeltchev K, et al. Comparison of medical treatment with percutaneous closure of patent foramen ovale in patients with cryptogenic stroke. J Am Coll Cardiol (in press)

9 Wilmshurst PT, Nightingale S, Walsh KP, et al. Effect on migraine of closure of cardiac right-to-left shunts to prevent recurrence of decompression illness or stroke or for hemodynamic reasons. Lancet 2000;356:1648-51.

10 Schwerzmann M, Windecker S, Nedeltchev K, et al. Percutaneous closure of patent foramen ovale reduces migraine frequency [abstract]. Eur Heart $J$ 2003;24:130.

11 Rodriguez CJ, Homma S, Sacco RL, et al. Race-ethnic differences in patent foramen ovale, atrial septal aneurysm, and right atrial anatomy among ischemic stroke patients. Stroke 2003;34:2097-102.

12 Wilmshurst PT, Pearson MJ, Nightingale S, et al. Inheritance of persistent foramen ovale and atrial septal defects and the relation to familial migraine with aura. Heart 2004:1315-20.

13 Di Tullio M, Sacco RL, Venketasubramanian N, et al. Comparison of diagnostic techniques for the detection of patent foramen ovale in stroke patients. Stroke 1993;24:1020-4

14 Billinger M, Vogel R, Gerber S, et al. A new screening method for the detection of patent foramen ovale in divers [abstract]. Kardiovask Med 2002;5:44S.

\section{IMAGES IN CARDIOLOGY}

\section{Seagulls flying in the echo lab}

A "seagull's cry murmur" is defined as a murmur imitating the cooing sound of a seagull. This type of murmur is typically characterised by a musical timbre and a high frequency, and may occur as a result of various valve diseases. It is usually described as a sign of tight calcific aortic stenosis, when the murmur's high frequency components are transmitted to the lower left sternal border and the cardiac apex during most of systole (Gallavardin's phenomenon). In this condition, the typical harsh timbre of the ejective murmur tends to assume a musical high pitched quality, resembling that of mitral regurgitation, which may be reminiscent of the cry of a seagull. A protodiastolic murmur with similar characteristics, typically in decrescendo, may occur in severe aortic valve regurgitation, particularly when the regurgitant flow presents high velocities. However, a seagull's cry murmur may also be the sign of mitral regurgitation or prolapse. Similarly, the musical and holosystolic sound reflects the presence of high frequency components due to high velocities of reflow. This image represents the pulsed Doppler pattern of mitral flow, obtained in a subject with mitral regurgitation. Curiously, in the context of this mitral regurgitation spectrum, as obtained by continuous Doppler, seagull's stylised images are reproduced. This appearance of multiple winged signs refers doi: $10.1136 /$ hrt. 2004.037200

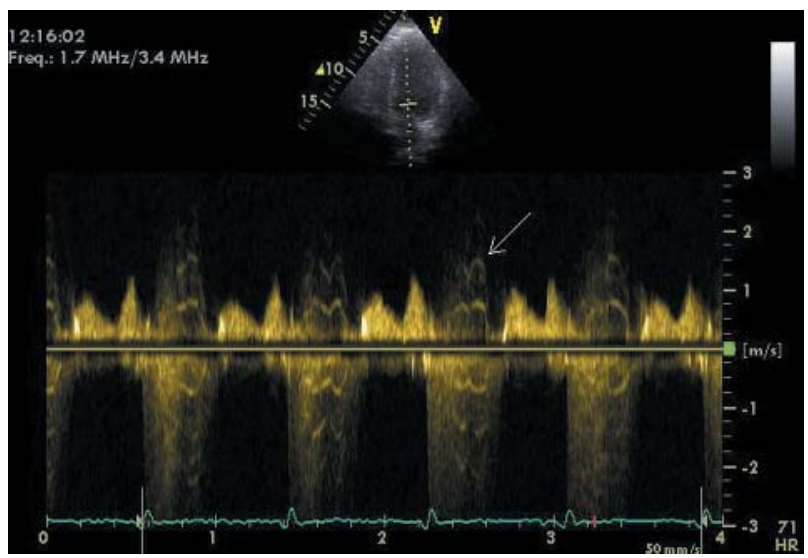

to the presence of high frequency components and harmonics that are likely to be caused by ruptured chordae tendinae.

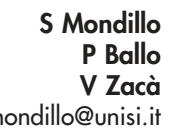

